The reason given by the manufacturers for the alternative sites recommended for children and adults is simply that these were the sites used in the trials of the vaccine (SmithKline Beecham, personal communication). The only indications for administration of hepatitis $B$ vaccine into the upper arm are thus convention and convenience.

Overweight adults generally have a problem with subcutaneous fat on the arms as well as the legs and, although the anterolateral aspect of the thigh may be more severely affected, the number of health service workers in whom this precludes an intramuscular injection is small. The anterolateral aspect of the thigh requires penetration of fascia lata to enter muscle and the feeling of "give" resulting from passage of the needle is a better indicator of the correct site for injection than anything available in the upper arm. It is also easier to differentiate between the buttock and anterolateral aspect of the thigh than between different sites over the deltoid and upper arm.

Work is currently in progress at the Communicable Diseases Surveillance Centre to identify whether any site of injection carries an increased risk of local reaction; the lack of accurate denominator data (numbers of children immunised at different sites) may make this difficult to complete (N Begg, personal communication). Any loss of skin continuity in the upper arm and deltoid region will, however, be more likely to scar badly and will definitely be more obvious than on the anterolateral aspect of the thigh. In an increasingly litiginous population the use of a site where any penetrating injury can result in unsightly scars or keloid formation with no apparent advantage to the patient must be questioned:

All patients should be advised of the alternative sites available for vaccination by intramuscular injection; many will prefer the upper arm but the choice should be theirs.

MARK HENLEY

North East Thames Regional Plastic Surgery Unit,

St Andrews Hospital,

Billericay,

Essex CM12 OBH

1 Payton C. Site of injection for vaccination. BMf 1992;305:364. (8 August.)

\section{Early treatment of meningococcal disease}

EDITOR,-We recently presented data from five health districts on the effect of early treatment with parenteral penicillin on mortality in suspected meningococcal disease..$^{1-3}$ Although in each district there was a lower mortality in those treated early, the reductions in mortality failed to reach significance. As case definitions in the studies were comparable we tested the effect of combining data on the 487 patients from the three published reports.

The overall case fatality was $9 \cdot 7 \%(47 / 487)$. A total of 358 patients did not receive parenteral penicillin before admission, of whom 41 (11.5\%) died; by comparison, six deaths $(4 \cdot 7 \%)$ occurred among the 129 patients who received parenteral penicillin before transfer to hospital. The risk of death in patients not given parenteral penicillin was significantly increased (Mantel-Haenszel weighted odds ratio $2.61 ; 95 \%$ confidence interval 1.04 to $7 \cdot 18)$. These data confirm the benefit of early antibiotic treatment.

The early diagnosis of meningococcal disease may present formidable problems in general practice. Individual doctors will see few cases in their professional lifetime, even in districts with a high incidence. Early symptoms are non-specific, and up to $40 \%$ of patients may not develop a rash in the period before admission to hospital. The diagnosis was, however, suspected in over three quarters of patients seen by a doctor before transfer to hospital in the Darlington study.' We encourage our general practitioner colleagues to carry benzylpenicillin at all times and to give it without hesitation if meningococcal disease is possible.

Public health physicians with special responsibility for communicable disease control can influence rates of giving penicillin. ${ }^{2}$ Departments of public health medicine are well placed to collaborate with microbiologists and hospital physicians to mount information and alerting campaigns directed at local family doctors to sustain awareness of meningococcal disease and to recommend early use of parenteral penicillin. Application of this strategy nationwide could have a considerable impact on the case fatality rate from meningococcal disease.

KEITH CARTWRIGHT

Public Health Laboratory,

Gloucestershire Royal Hospital,

Gloucester GL1 3NN

North Yorkshire Health Project,

JEFFRIE STRANG

York YO3 $4 \mathrm{XF}$

SAVITA GOSSAIN

Department of Microbiology

Worcester Royal Infirmary,

Worcester WR1 3AS

NORMAN BEGG

Public Health Laboratory Service

Communicable Disease Surveillance Centre,

London NW9 5EQ

1 Strang JR, Pugh EJ. Meningococcal infections: reducing the case fatality rate by giving penicillin before admission to hospital. BMF 1992;305:141-3. (18 July.)

2 Cartwright $K$, Reilly $S$, White D, Stuart J. Early treatment with parenteral penicillin in meningococcal disease. BMF 1992;305: 143-7. (18 July.)

3 Gossain S, Constantine CE, Webberley JM. Early parenteral penicillin in meningococcal disease. $B \mathcal{F}^{\mathcal{f}}$ 1992;305:523-4. (29 August.)

EDITOR,-Two recent papers have analysed the relation between antibiotic treatment given before admission to hospital and mortality from meningococcal disease and found a trend with fewer deaths among patients receiving parenteral antibiotics before admission. ${ }^{12}$

We have just made a historical cohort study including all 177 patients who were admitted with meningococcal disease to hospitals in the county of North Jutland, Denmark, between 1 January 1980 and 31 December $1989 .^{3}$ As part of this investigation we analysed the relation between prehospital parenteral antibiotic treatment and death from meningococcal disease.

The standard recommendations of the Danish health authorities regarding prehospital treatment of meningococcal disease are that penicillin $G$ should be given intravenously (or intramuscularly if the intravenous attempt is not immediately successful) when petechiae or other signs of meningococcal sepsis are present, and if the patient shows signs of severe meningitis and the transport time to the nearest hospital is more than half an hour.

When the general practitioner suspected meningitis (in 123 of the 177 patients) the recommendations for immediate antibiotic treatment should have been followed. Of these 123 patients, 98 should have had parenteral antibiotic treatment before admission to hospital. In fact only 25 had treatment according to recommendations.

Of the 98 patients, 10 died: six of the 25 patients who received parenteral antibiotics and four of the 73 patients who did not receive parenteral antibiotics before admission. The higher mortality in the parenteral antibiotic group is statistically significant $(p=0.03$, Fisher's test) with odds ratio of $5.4(95 \%$ confidence interval 1.5 to $19 \cdot 2)$.

Of the 177 patients in the study, a total of 11 died: six of 25 patients receiving parenteral antibiotic before admission and five of 152 not receiving parenteral antibiotics before admission. The difference in mortality is statistically significant with odds ratio of $9 \cdot 3(3 \cdot 1$ to $27 \cdot 9)$

Overall 11 patients died in our study. Ten of 15 patients with disseminated intravascular coagulation died, whereas only one patient died out of 162 without disseminated intravascular coagulation. Using logistic regression analysis on the whole study population we found that only the presence of disseminated intravascular coagulation predicted death with statistical significance $(p<0.001)$ with an odds ratio for death of $170(15$ to 1930).

The high mortality (24\%) among the patients who received prehospital treatment with parenteral antibiotics was probably due to a particularly fulminant infection, easily recognisable at home. Thus, our study shows the opposite tendency to the two British investigations. To further elucidate the role of parenteral prehospital antibiotic treatment it seems necessary to evaluate the severity of the meningococcal disease at the time of decision to give the treatment. We recommend stratified analysis taking this into account in future investigations.

Department of Medicine $\mathrm{V}$

University Hospital of Aarhus,

DK-8000 Aarhus C

Denmark

Department of Internal Medicine,

Randers Hospital,

DK-8900 Randers

HENRIK TOFT SøRENSEN

JENS MøLLER-PETERSEN

HENRIK BYGUM KRARUP HELENA PEDERSEN HELLE HANSEN

Department of Internal Medicine $M$

Aalborg Hospital,

DK-9000 Aalborg

Department of Public Health,

County of North Jutland,

DK-9000 Aalborg

1 Strang JR, Pugh EJ. Meningococcal infections: reducing the case fatality rate by giving penicillin before admission to hospital. BMF 1992;305: 141-3. (18 July.)

2 Cartwright K, Reilly S, White D, Stuart J. Early treatment with parenteral penicillin in meningococcal disease. BMf 1992; 305:143-7.

3 Sørensen HT, Møller-Petersen J, Krarup HB, Pedersen H, Hansen H, Hamburger H. Diagnostic problems with meningococcal disease in general practice. $f$ Clin Epidemio (in press)

4 [The State Serum Institute: meningococcal disease/purulen meningitis.] EpiNyt 1984:week 32. (In Danish.)

\section{Controlling leprosy}

EdIToR,-Christian Lienhardt and P E M Fine do not believe that using multidrug treatment alone will achieve the World Health Assembly's goal of eliminating leprosy as a public health problem by the year 2000.' They do accept, however, that "dramatic falls in the incidence of leprosy (or case detection rates) have been documented [which] began in most countries long before the introduction of multidrug treatment" but attribute this decline to "improving socioeconomic standards" and $B C G$ vaccination. ${ }^{1}$ They ignore completely the effect of dapsone monotherapy in interrupting transmission despite evidence from northern Nigeria, Uganda, India, and central Burma.

In striking contrast, none of the patients in Karimui, New Guinea, originally received any chemotherapy and the incidence of the disease remained constant at 5/1000 from 1964 to $1969{ }^{3} \mathrm{~A}$ BCG vaccine trial started there in 1964; the final results were expressed in incidence per 1000 person years ${ }^{4}$ and the significance of the findings has been missed, In November 1967 chemotherapy was started with acedapsone, a long acting sulphone. ${ }^{3}$ Incidence rates can be calculated in vaccinated and unvaccinated groups from the cumulative totals previously published, ${ }^{45}$ and the considerable decline in unvaccinated groups (figure) must be attributable to chemotherapy. Furthermore, only one new patient in the unvaccinated group ${ }^{45}$ was detected after 1972 with 